\title{
GAMBLING TEAMS AND WAITING TIMES FOR PATTERNS IN TWO-STATE MARKOV CHAINS
}

\author{
JOSEPH GLAZ, ${ }^{*}$ University of Connecticut \\ MARTIN KULLDORFF, ${ }^{* *}$ Harvard Medical School and Harvard Pilgrim Health Care \\ VLADIMIR POZDNYAKOV, ${ }^{* * * *}$ University of Connecticut \\ J. MICHAEL STEELE, ${ }^{* * * *}$ University of Pennsylvania
}

\begin{abstract}
Methods using gambling teams and martingales are developed and applied to find formulae for the expected value and the generating function of the waiting time to observation of an element of a finite collection of patterns in a sequence generated by a two-state Markov chain of first, or higher, order.
\end{abstract}

Keywords: Gambling; team; waiting time; pattern; success run; failure run; Markov chain; martingale; stopping time; generating function

2000 Mathematics Subject Classification: Primary 60J10

Secondary 60G42

\section{Introduction}

How long must we observe a stochastic process with values from a finite alphabet until we see a realization of a pattern that belongs to a specified collection, $\mathcal{C}$, of possible patterns? For independent processes this is an old question; in some special cases it was even considered by Feller (1968, pp. 326-328). Nevertheless, in the context of more general processes, or even for Markov chains, there are many natural problems that have not been fully addressed. The main goal here is to show how some progress can be made by further developing the martingale methods that were introduced by $\mathrm{Li}$ (1980) and Gerber and $\mathrm{Li}$ (1981) in their investigation of independent sequences. Their key observation was that information on the occurrence times of patterns can be obtained from the values assumed by a specially constructed auxiliary martingale at a certain well-chosen time.

In the case of Markov chains of first, or higher, order, this observation is still useful, but to utilize it requires a rather more elaborate plan for the construction of the auxiliary martingale. This construction depends in turn on several general devices that seem more broadly useful; these include 'teams of gamblers,' 'watching then betting,' 'reward matching,' and a couple of other devices that will be described shortly.

Before giving that description, we should note that pattern matching has been studied using many other techniques. The combinatorial methods of Guibas and Odlyzko (1981a), (1981b)

\footnotetext{
Received 4 April 2003; revision received 14 November 2005.

* Postal address: Department of Statistics, University of Connecticut, 215 Glenbrook Road, U-4120, Storrs, CT 06269-4120, USA.

** Postal address: Department of Ambulatory Care and Prevention, Harvard Medical School, 133 Brookline Avenue, Boston, MA 02215-3920, USA.

*** Email address: vladimir.pozdnyakov@uconn.edu

**** Postal address: Department of Statistics, Wharton School, Huntsman Hall 447, University of Pennsylvania, Philadelphia, PA 19104, USA.
} 
are particularly effective, and there are numerous treatments of pattern matching problems using probabilistic techniques, such as Benevento (1984), Biggins and Cannings (1987a), (1987b), Blom and Thorburn (1982), Breen et al. (1985), Chryssaphinou and Papastavridis (1990), Han and Hirano (2003), Pozdnyakov et al. (2005), Pozdnyakov and Kulldorff (2006), Robin and Daudin (1999), Stefanov (2003), and Uchida (1998). One of the more general techniques is the Markov chain embedding method introduced by Fu (1986), which has been further developed by Antzoulakos (2001), Fu (2001), Fu and Chang (2002), and Fu and Koutras (1994). The approach of Stefanov (2000) and Stefanov and Pakes (1997) also uses Markov chain embedding, though their method differs substantially from that of Fu. Only a few investigations have considered waiting time problems for higher-order Markov chains, and these have all focused on specific waiting times such as the 'sooner or later' problem studied by Aki et al. (1996).

\section{Expected waiting time until a pattern is observed}

We take $\left\{Z_{n}, n \geq 1\right\}$ to be a Markov chain with two states, $S$ and $F$, which may model 'success' and 'failure.' We suppose the chain to have the initial distribution with $\mathrm{P}\left(Z_{1}=S\right)=$ $p_{S}$ and $\mathrm{P}\left(Z_{1}=F\right)=p_{F}$, and the transition matrix

$$
\left(\begin{array}{ll}
p_{S S} & p_{F S} \\
p_{S F} & p_{F F}
\end{array}\right),
$$

where $p_{S F}$ is shorthand for $\mathrm{P}\left(Z_{n+1}=F \mid Z_{n}=S\right)$. We then consider a collection, $\mathcal{C}$, of finite sequences $A_{i}, 1 \leq i \leq K$, from the two-letter alphabet $\{S, F\}$. If $\tau_{A_{i}}$ denotes the time elapsed before the pattern $A_{i}$ is first observed as a completed run in the series $Z_{1}, Z_{2}, \ldots$, then the random variable of main interest here is $\tau_{\mathcal{C}}=\min \left\{\tau_{A_{1}}, \ldots, \tau_{A_{K}}\right\}$, the time at which we first observe a pattern from $\mathcal{C}$. Throughout our discussion we assume that no pattern from $\mathcal{C}$ contains another pattern from $\mathcal{C}$ as an initial segment. Naturally, this assumption entails no loss of generality.

\subsection{A run of 'failures' under a Markov model}

To illustrate the construction, we first consider the rather easy case in which $K=1$ and the pattern $A_{1}$ is a run of $r$ consecutive $F$ s. Thus, we will compute the expected value of $\tau=\tau_{A_{1}}$, the time of the first completion of a run of $r$ 'failures' under our two-state Markov model. This example can be treated in several ways, and offers a useful benchmark for more challenging examples.

We consider a casino where gamblers may bet in successive rounds on the output of our given two-state Markov chain, and assume that the casino is fair in a sense that we will soon make precise. We then consider a sequence of gamblers, one of whom arrives just before each new round of betting. Thus, gambler $n+1$ arrives in time to observe the result of the $n$th trial, $Z_{n}$, and we assume that he bets a dollar on the event that the next trial yields an $F$. If $Z_{n+1}=S$, he loses his dollar and leaves the game. If he is lucky, and $Z_{n+1}=F$, then he wins $1 / p_{S F}$ when $Z_{n}=S$ and wins $1 / p_{F F}$ when $Z_{n}=F$. This is the sense in which the casino is fair; the expected return on a one-dollar bet is one dollar.

After this gambler collects his money, he then bets his entire capital on the event that $Z_{n+2}=F$. Again, if $Z_{n+2}=S$ then the gambler leaves the game with nothing, while if $Z_{n+2}=F$ then the gambler wins this round and his capital is increased by a factor $1 / p_{F F}$. Successive rounds proceed in the same way, with a new gambler arriving at each new round and with the gamblers from earlier periods either continuing to win or else going broke and leaving. 
We need to be precise about how this process ends. If gambler $n+1$ begins by observing $Z_{n}=S$, then he bets until he either goes broke or observes $r$ successive $F$ s, and if he begins by observing $Z_{n}=F$, then he bets until he either goes broke or observes $r-1$ successive $F$ s. Once some gambler stops without going broke, all of the gambling stops.

Finally, we let $X_{n}$ denote the casino's net gain at the conclusion of round $n$. Since each bet is fair and since the bet sizes depend only on the previous observations, the sequence $X_{n}$ is a martingale with respect to the $\sigma$-field generated by $\left\{Z_{n}, n \geq 1\right\}$. Now, to obtain a relation that will determine $\mathrm{E}[\tau]$, we just need to consider the casino's net gain when the gambling stops, $X_{\tau}$. By calculating $\mathrm{E}\left[X_{\tau}\right]$ in two ways we will then obtain the expected value of the time $\tau$.

At time $\tau$, many gamblers are likely to have lost all their money; only those who entered the game after round number $\tau-r-1$ have any money. We thus face two different ending scenarios. First, it could happen that we have a block (denoted by $F^{(r)}$ ) of $r$ instances of $F$ that occurs at the very beginning of the sequence $\left\{Z_{n}, n \geq 1\right\}$. Second, it could happen that we end with $S F^{(r)}$, an $S$ followed by a block of $r$ instances of $F$. Obviously we do not need to consider the possibility of ending with $F F^{(r)}=F^{(r+1)}$, since by definition $F^{(r)}$ cannot occur before time $\tau$.

When we sum the wins and losses of all of the gamblers, we then find that the value of the stopped martingale $X_{\tau}$ is exactly given by

$$
X_{\tau}= \begin{cases}\tau-1-\frac{1}{p_{F F}^{r-1}}-\frac{1}{p_{F F}^{r-2}}-\cdots-\frac{1}{p_{F F}} & \text { in the first scenario, } \\ \tau-1-\frac{1}{p_{S F} p_{F F}^{r-1}}-\frac{1}{p_{F F}^{r-1}}-\frac{1}{p_{F F}^{r-2}}-\cdots-\frac{1}{p_{F F}} & \text { in the second scenario, }\end{cases}
$$

which can be written more succinctly as

$$
X_{\tau}= \begin{cases}\tau-1-\frac{1-p_{F F}^{r-1}}{p_{F F}^{r-1}\left(1-p_{F F}\right)} & \text { in the first scenario, } \\ \tau-1-\frac{1}{p_{S F} p_{F F}^{r-1}}-\frac{1-p_{F F}^{r-1}}{p_{F F}^{r-1}\left(1-p_{F F}\right)} & \text { in the second scenario. }\end{cases}
$$

Since $\mathrm{E}[\tau]<\infty$ and the increments of $X_{n}$ are bounded, the optional stopping theorem for martingales (see, e.g. Williams (1991, p. 100)) tells us that $0=\mathrm{E}\left[X_{1}\right]=\mathrm{E}\left[X_{\tau}\right]$. From this identity and the formula for $X_{\tau}$, algebraic simplification gives us

$$
\mathrm{E}[\tau]=1+p_{F} \frac{1-p_{F F}^{r-1}}{1-p_{F F}}+\left(1-p_{F} p_{F F}^{r-1}\right)\left(\frac{1}{p_{S F} p_{F F}^{r-1}}+\frac{1-p_{F F}^{r-1}}{p_{F S} p_{F F}^{r-1}}\right) .
$$

\subsection{Second step: a single pattern}

We now consider the more subtle case of a single (nonrun) pattern $A$ of length $r$, and for specificity we assume that the pattern begins with an $F$; hence, $A=F B$ with $B \in\{S, F\}^{r-1}$. As before, we consider a sequence of gamblers, but this time we need to consider three different ending scenarios:

1. $A$ occurs at the beginning of the sequence $\left\{Z_{n}, n \geq 1\right\}$.

2. The pattern $S A$ occurs.

3. The pattern $F A$ occurs. 
The probability, $p_{1}$, of the first scenario is trivial to compute, but we then face a problem. We do not know the probability of the pattern $S A$ appearing earlier than the pattern $F A$, so the probabilities of the second and third ending scenarios are not readily computable. To circumvent this problem, we introduce two teams of gamblers.

\subsection{Rules for the gambling teams}

1. A gambler from the first team who arrives before round $n$ watches the result of the $n$th trial and then bets $y_{1}$ dollars on the first letter in the sequence $A$. If he wins he then bets all of his capital on the next letter in the sequence $A$, and continues in this way until he either loses his capital or observes all of the letters of $A$. Such players are called 'straightforward gamblers.'

2. The gamblers of the second team make use of the information that they gather. On the one hand, if gambler $n+1$ observes that $Z_{n}=S$ just before he begins his play, then he bets like a straightforward gambler, except that he begins by wagering $y_{2}$ dollars on the first letter of pattern $A$. On the other hand, if he observes that $Z_{n}=F$ when he first arrives, then he wagers $y_{2}$ dollars on the first letter of the pattern $B$. He then continues to wager on the successive letters of $B$ until either he loses or he observes $B$. Such players are called 'smart gamblers.'

The two gambling teams continue their betting until one team stops. At that time, all gambling stops and we consider the wins and losses. Only those gamblers who entered the game after the time $\tau-r-1$ will have any money, and the amount they have will depend on the ending scenario. If we let $W_{i j} y_{j}$ denote the amount of money that team $j \in\{1,2\}$ wins in scenario $i \in\{1,2,3\}$, then the values $W_{i j}$ are easy to compute and, in terms of these values, the stopped martingale $X_{\tau}$, which represents the casino's net gain, is given by

$$
X_{\tau}= \begin{cases}\left(y_{1}+y_{2}\right)(\tau-1)-y_{1} W_{11}-y_{2} W_{12} & \text { in the first scenario, } \\ \left(y_{1}+y_{2}\right)(\tau-1)-y_{1} W_{21}-y_{2} W_{22} & \text { in the second scenario, } \\ \left(y_{1}+y_{2}\right)(\tau-1)-y_{1} W_{31}-y_{2} W_{32} & \text { in the third scenario. }\end{cases}
$$

Now, if we take $\left(y_{1}^{*}, y_{2}^{*}\right)$ to be a solution to the system

$$
y_{1}^{*} W_{21}+y_{2}^{*} W_{22}=1, \quad y_{1}^{*} W_{31}+y_{2}^{*} W_{32}=1,
$$

we see that, with these bet sizes, we have a very simple formula for $X_{\tau}$ :

$$
X_{\tau}= \begin{cases}\left(y_{1}^{*}+y_{2}^{*}\right)(\tau-1)-y_{1}^{*} W_{11}-y_{2}^{*} W_{12} & \text { in the first scenario, } \\ \left(y_{1}^{*}+y_{2}^{*}\right)(\tau-1)-1 & \text { in the second scenario, } \\ \left(y_{1}^{*}+y_{2}^{*}\right)(\tau-1)-1 & \text { in the third scenario. }\end{cases}
$$

The optional stopping theorem then gives us

$$
0=\left(y_{1}^{*}+y_{2}^{*}\right)(\mathrm{E}[\tau]-1)-p_{1}\left(y_{1}^{*} W_{11}+y_{2}^{*} W_{12}\right)-\left(1-p_{1}\right),
$$

where $p_{1}$ is the probability of scenario one occurring. We therefore find that

$$
\mathrm{E}[\tau]=1+\frac{p_{1}\left(y_{1}^{*} W_{11}+y_{2}^{*} W_{12}\right)+1-p_{1}}{y_{1}^{*}+y_{2}^{*}} .
$$

Formula (1) is more explicit than it may at first appear. In the typical case, the calculation of $p_{1},\left\{W_{i j}, 1 \leq i \leq 3,1 \leq j \leq 2\right\}$, and $\left\{y_{j}^{*}, 1 \leq j \leq 2\right\}$ is genuinely routine, as can be seen in the following example. 


\subsection{Example: waiting time until FSF is observed}

Here, our straightforward gamblers bet $y_{1}$ dollars on FSF without regard for the preceding observation, while the smart gamblers bet $y_{2}$ dollars on $F S F$ if they observed $S$ before placing their first bet, and bet $y_{2}$ dollars on $S F$ if they observed $F$. The three ending scenarios are now the occurrence of $F S F$ at the beginning (scenario one), the occurrence of SFSF at the end (scenario two), or the occurrence of $F F S F$ at the end (scenario three). The $3 \times 2$ 'profit matrix' $\left\{W_{i j}\right\}$ is then given by

$$
\left(\begin{array}{cc}
\frac{1}{p_{S F}} & \frac{1}{p_{F S} p_{S F}}+\frac{1}{p_{S F}} \\
\frac{1}{p_{S F} p_{F S} p_{S F}}+\frac{1}{p_{S F}} & \frac{1}{p_{S F} p_{F S} p_{S F}}+\frac{1}{p_{F S} p_{S F}}+\frac{1}{p_{S F}} \\
\frac{1}{p_{F F} p_{F S} p_{S F}}+\frac{1}{p_{S F}} & \frac{1}{p_{F S} p_{S F}}+\frac{1}{p_{S F}}
\end{array}\right)
$$

and the bet sizes $y_{1}^{*}$ and $y_{2}^{*}$ are determined by the relations

$$
\begin{array}{r}
y_{1}^{*}\left(\frac{1}{p_{S F} p_{F S} p_{S F}}+\frac{1}{p_{S F}}\right)+y_{2}^{*}\left(\frac{1}{p_{S F} p_{F S} p_{S F}}+\frac{1}{p_{F S} p_{S F}}+\frac{1}{p_{S F}}\right)=1, \\
y_{1}^{*}\left(\frac{1}{p_{F F} p_{F S} p_{S F}}+\frac{1}{p_{S F}}\right)+y_{2}^{*}\left(\frac{1}{p_{F S} p_{S F}}+\frac{1}{p_{S F}}\right)=1,
\end{array}
$$

which can be solved to obtain

$$
y_{1}^{*}=\frac{p_{F F} p_{F S} p_{S F}}{p_{F S}+p_{S F}+p_{F S} p_{S F}} \quad \text { and } \quad y_{2}^{*}=\frac{p_{F S} p_{S F}\left(p_{S F}-p_{F F}\right)}{p_{F S}+p_{S F}+p_{F S} p_{S F}} .
$$

The probability, $p_{1}$, of the first scenario is just $p_{F} p_{F S} p_{S F}$, so after substitution and simplification the general formula (1) yields

$$
\mathrm{E}\left[\tau_{F S F}\right]=1+\frac{p_{S}}{p_{S F}}+\frac{1}{p_{S F}^{2}}+\frac{1}{p_{F S} p_{S F}},
$$

which is as explicit as could be desired.

\section{Expected time until observing one of many patterns}

We now consider a collection, $\mathcal{C}=\left\{A_{i}, 1 \leq i \leq K\right\}$, of $K$ strings of possibly varying lengths from the two-letter alphabet $\{S, F\}$, and we take on the task of computing the expected value of $\tau_{\mathcal{C}}=\min \left\{\tau_{A_{1}}, \ldots, \tau_{A_{K}}\right\}$, the time at which any one of the patterns in $\mathcal{C}$ is first observed. The method we propose is analogous to the two-team method we used in the previous section, although many teams are now needed. The real challenge is the construction of the list of appropriate ending scenarios, which now requires some algorithmic techniques.

\subsection{Listing the ending scenarios}

Given the collection $\mathcal{C}=\left\{A_{i}\right\}_{1 \leq i \leq K}$, we first consider the set sequence transformation

$$
\mathcal{C}=\left\{A_{i}\right\}_{1 \leq i \leq K} \rightarrow\left\{S A_{i}, F A_{i}\right\}_{1 \leq i \leq K}=\left\{B_{i}\right\}_{1 \leq i \leq 2 K}=\mathcal{C}^{\prime},
$$


which doubles the cardinality of $\mathcal{C}$. We then delete from $\mathcal{C}^{\prime}$ each pattern $B$ that can occur only after the stopping time $\tau_{\mathcal{C}}$. The resulting collection, $\mathcal{C}^{\prime \prime}$, is called the 'final list.' We denote the elements of $\mathcal{C}^{\prime \prime}$ by $C_{i}, 1 \leq i \leq K^{\prime}$, and note that $K \leq K^{\prime} \leq 2 K$.

To illustrate the construction, suppose that the initial collection is $\mathcal{C}=\{F S F, F F\}$. The 'doubling step' (from $\mathcal{C}$ to $\mathcal{C}^{\prime}$ ) gives us $\mathcal{C}^{\prime}=\{S F S F, F F S F, S F F, F F F\}$. Since $F F S$ and $F F F$ cannot occur before $\tau_{\mathcal{C}}$, these are eliminated from $\mathcal{C}^{\prime}$ and the final list is simply $\mathcal{C}^{\prime \prime}=\{S F S F, S F F\}$. Similarly, if the initial collection is $\mathcal{C}=\{F S, S S S\}$, then the final list is $\mathcal{C}^{\prime \prime}=\{S F S, F F S\}$.

Now, before we describe the ending scenarios, we need one further notion. If patterns $C$ and $C^{\prime}$ in the final list $\mathcal{C}^{\prime \prime}$ satisfy $C=S A$ and $C^{\prime}=F A$ for some pattern $A \in \mathcal{C}$, then we say that $C$ and $C^{\prime}$ are 'matched.' Also, if $C$ and $C^{\prime}$ are matched and $C=S A$ and $C^{\prime}=F A$, then we say that $C$ and $C^{\prime}$ are 'generated' by $A$. Finally, even though there are many ending scenarios, they may be classified into three basic kinds.

1. There are $K$ scenarios in which we observe an element of $\mathcal{C}$ to be an initial segment of the Markov sequence $\left\{Z_{n}, n \geq 1\right\}$.

2. There is a scenario for each unmatched pattern from $\mathcal{C}^{\prime \prime}$. We denote the number of these by $L$.

3. There is pair of scenarios for each matched pattern from $\mathcal{C}^{\prime \prime}$. We denote the number of these by $2 M$.

\subsection{From the listing to the teams}

For each scenario associated with the unmatched pattern $C_{j}$, we introduce one team of straightforward gamblers who bet $y_{j}$ dollars on the pattern $A_{i}$ that generated $C_{j}$. For each pair of scenarios associated with the matched patterns $C_{p}$ and $C_{m}$, which were generated by pattern $A_{k}$, say, we introduce two teams. One team bets $y_{p}$ dollars on $A_{k}$ in the straightforward way, and the other bets $y_{m}$ dollars on $A_{k}$ in the smart way. If $W_{i j} y_{j}, i=1,2, \ldots, K+L+2 M$, $j=1,2, \ldots, L+2 M$, denotes the amount of money that the $j$ th team wins in the $j$ th scenario, then the stopped martingale $X_{\tau}$ is given by the sum

$$
X_{\tau}=\sum_{j=1}^{L+2 M} y_{j}(\tau-1)-S\left(y_{1}, \ldots, y_{L+2 M}\right),
$$

where

$$
S\left(y_{1}, \ldots, y_{L+2 M}\right)=\sum_{i=1}^{K+L+2 M} \mathbf{1}_{E_{i}} \sum_{j=1}^{L+2 M} y_{j} W_{i j} .
$$

Here, $\mathbf{1}_{E_{i}}$ is the indicator function for the event, $E_{i}$, that the $i$ th scenario occurs.

If $\left(y_{1}^{*}, \ldots, y_{L+2 M}^{*}\right)$ is a solution to the linear system

$$
\begin{aligned}
y_{1}^{*} W_{K+1,1}+\cdots+y_{L+2 M}^{*} W_{K+1, L+2 M} & =1, \\
\vdots & \vdots \\
y_{1}^{*} W_{K+L+2 M, 1}+\cdots+y_{L+2 M}^{*} W_{K+L+2 M, L+2 M} & =1,
\end{aligned}
$$


then we have

$$
S\left(y_{1}^{*}, \ldots, y_{L+2 M}^{*}\right)= \begin{cases}\sum_{j=1}^{L+2 M} y_{j}^{*} W_{i j} & \text { in scenario } i \in\{1,2, \ldots, K\} \\ 1, & \text { in scenario } i>K .\end{cases}
$$

By the optional stopping theorem, we have

$$
0=\mathrm{E}\left[X_{1}\right]=\mathrm{E}\left[X_{\tau_{\mathcal{C}}}\right]=\sum_{j=1}^{L+2 M} y_{j}^{*}\left(\mathrm{E}\left[\tau_{\mathcal{C}}\right]-1\right)-\sum_{i=1}^{K} p_{i} \sum_{j=1}^{L+2 M} y_{j}^{*} W_{i j}-\left(1-\sum_{i=1}^{K} p_{i}\right),
$$

where $p_{i}$ is the probability that $A_{i}$ is an initial segment of $\left\{Z_{n}, n \geq 1\right\}$. We can now solve this equation to obtain a formula for $E\left[\tau_{\mathcal{C}}\right]$, which we summarize as a theorem as follows.

Theorem 1. If $\left(y_{1}^{*}, y_{2}^{*}, \ldots, y_{L+2 M}^{*}\right)$ solves the linear system (2), then

$$
\mathrm{E}\left[\tau_{\mathcal{C}}\right]=1+\frac{\sum_{i=1}^{K} p_{i} \sum_{j=1}^{L+2 M} y_{j}^{*} W_{i j}+\left(1-\sum_{i=1}^{K} p_{i}\right)}{\sum_{j=1}^{L+2 M} y_{j}^{*}} .
$$

As before, this formula is more explicit than it may at first appear. For example, all of the required terms can be computed straightforwardly in problems of reasonable size.

\subsection{Computation of the profit matrix}

Formula (3) requires the computation of the profit matrix $\left\{W_{i j}\right\}$. We will first show how this can be done in general. The method will then be applied to a specific example to confirm that (3) may be rewritten in terms of the basic model parameters.

Consider a scenario that ends with pattern $C=c_{1} c_{2} \cdots c_{m} \in \mathcal{C}^{\prime \prime}$. The team of straightforward gamblers who begin by betting one dollar and continue by betting on the successive terms of the pattern $A=a_{1} a_{2} \cdots a_{p}$ will, by time $\tau_{\mathcal{C}}$, have won an amount

$$
\sum_{i=1}^{\min \{m-1, p\}} \delta_{i}^{\mathrm{st}}(A, C)
$$

where

$$
\delta_{i}^{\mathrm{st}}(A, C)= \begin{cases}\frac{1}{p_{c_{m-i} a_{1}} p_{a_{1} a_{2}} \cdots p_{a_{i-1} a_{i}}} & \text { if } a_{1}=c_{m-i+1}, a_{2}=c_{m-i+2}, \ldots, a_{i}=c_{m}, \\ 0 & \text { otherwise. }\end{cases}
$$

Similarly, in the same time, the team of smart gamblers will have won an amount

$$
\sum_{i=1}^{\min \{m-1, p\}} \delta_{i}^{\mathrm{sm} 1}(A, C)+\sum_{i=1}^{\min \{m-1, p-1\}} \delta_{i}^{\mathrm{sm} 2}(A, C)
$$


where

$$
\begin{aligned}
& \delta_{i}^{\mathrm{sm} 1}(A, C)= \begin{cases}\frac{1}{p_{c_{m-i} a_{1}} p_{a_{1} a_{2}} \cdots p_{a_{i-1} a_{i}}} & \text { if } a_{1}=c_{m-i+1}, a_{2}=c_{m-i+2}, \ldots, a_{i}=c_{m}, \\
0 & \text { and } c_{m-i} \neq a_{1}, \\
0 & \text { otherwise, }\end{cases} \\
& \delta_{i}^{\mathrm{sm} 2}(A, C)= \begin{cases}\frac{1}{p_{a_{1} a_{2}} p_{a_{2} a_{3}} \cdots p_{a_{i} a_{i+1}}} & \text { if } a_{1}=c_{m-i}, a_{2}=c_{m-i+1}, \ldots, a_{i+1}=c_{m}, \\
0 & \text { otherwise. }\end{cases}
\end{aligned}
$$

\subsection{Explicit determination of $E\left[\tau_{\mathcal{C}}\right]$}

To illustrate the use of (3), we consider the collection $\mathcal{C}=\{S S, F S F\}$. After doubling and elimination we obtain the final list $\{F S S, S F S F, F F S F\}$. We then need to work out the set of scenarios: we have two scenarios in which either $C_{1}=S S$ or $C_{2}=F S F$ occurs as an initial segment of $\left\{Z_{n}, n \geq 1\right\}$; we have the unmatched scenario $C_{3}=F S S$, associated with the pattern $S S$; and we have a pair of matched scenarios, $C_{4}=S F S F$ and $C_{5}=F F S F$, which are associated with the pattern $F S F$. The profit matrix $\left\{W_{i j}\right\}$ is then given by

$$
\left(\begin{array}{ccc}
\frac{1}{p_{S S}} & 0 & 0 \\
0 & \frac{1}{p_{S F}} & \frac{1}{p_{F S} p_{S F}}+\frac{1}{p_{S F}} \\
\frac{1}{p_{F S} p_{S S}}+\frac{1}{p_{S S}} & 0 & 0 \\
0 & \frac{1}{p_{S F} p_{F S} p_{S F}}+\frac{1}{p_{S F}} & \frac{1}{p_{S F} p_{F S} p_{S F}}+\frac{1}{p_{F S} p_{S F}}+\frac{1}{p_{S F}} \\
0 & \frac{1}{p_{F F} p_{F S} p_{S F}}+\frac{1}{p_{S F}} & \frac{1}{p_{F S} p_{S F}}+\frac{1}{p_{S F}}
\end{array}\right),
$$

and, after solving the corresponding linear system, we find that the appropriate initial team bets are given by

$$
y_{1}^{*}=\frac{p_{F S} p_{S S}}{1+p_{F S}}, \quad y_{2}^{*}=\frac{p_{F F} p_{F S} p_{S F}}{p_{F S}+p_{S F}+p_{F S} p_{S F}}, \quad y_{3}^{*}=\frac{p_{F S} p_{S F}\left(p_{S F}-p_{F F}\right)}{p_{F S}+p_{S F}+p_{F S} p_{S F}} .
$$

The probabilities, $p_{1}$ and $p_{2}$, that $S S$ and $F S F$ are initial segments of the process $\left\{Z_{n}, n \geq 1\right\}$ are respectively given by $p_{S} p_{S S}$ and $p_{F} p_{F S} p_{S F}$. Thus, (3) leads to the pleasantly succinct result

$$
\mathrm{E}\left[\tau_{\mathcal{C}}\right]=2+p_{S} p_{S F}+\frac{1-p_{S} p_{S S}}{p_{F S}} .
$$

\section{Generating functions for pattern waiting times}

To find the generating function of the waiting time $\tau_{\mathcal{C}}$, we need to introduce the same scenarios and the same betting teams, but we need to make some changes to the design of the initial bets. A gambler from the $j$ th team who arrives at moment $k-1$ and who begins his betting in round $k$ will now begin with a bet of size $y_{j} \alpha^{k}$, where $0<\alpha<1$. If $\alpha^{\tau_{\mathcal{C}}} W_{i j}(\alpha) y_{j}$ denotes the total winnings of the $j$ th team when the game ends in the $j$ th scenario, then we call 
$W_{i j}(\alpha)$ the ' $\alpha$-profit matrix.' As before, the $\alpha$-profit matrix does not depend on $\tau_{\mathcal{C}}$, and can be computed if we know the ending scenario.

If $X_{n}$ again denotes the casino's net gain at time $n$, then

$$
X_{\tau_{\mathcal{C}}}=\frac{\alpha^{2}-\alpha \alpha^{\tau}}{1-\alpha} \sum_{j=1}^{L+2 M} y_{j}-S\left(\alpha, y_{1}, \ldots, y_{L+2 M}\right),
$$

where

$$
S\left(\alpha, y_{1}, \ldots, y_{L+2 M}\right)=\sum_{i=1}^{K+L+2 M} \mathbf{1}_{E_{i}} \sum_{j=1}^{K+2 M} \alpha^{\tau} y_{j} W_{i j}(\alpha) .
$$

As before, $\mathbf{1}_{E_{i}}$ is the indicator function for the event, $E_{i}$, that the $j$ th scenario occurs.

If $\left(y_{1}^{*}, \ldots, y_{L+2 M}^{*}\right)$ is a solution to the linear system

$$
\begin{aligned}
y_{1}^{*} W_{K+1,1}(\alpha)+\cdots+y_{L+2 M}^{*} W_{K+1, L+2 M}(\alpha) & =1, \\
\vdots & \vdots \\
y_{1}^{*} W_{K+L+2 M, 1}(\alpha)+\cdots+y_{L+2 M}^{*} W_{K+L+2 M, L+2 M}(\alpha) & =1,
\end{aligned}
$$

then we might hope to mimic our earlier calculation of $\mathrm{E}\left[X_{\tau_{\mathcal{C}}}\right]$, but unfortunately we run into trouble since $\mathrm{E}\left[\alpha^{\tau_{\mathcal{C}}} \mathbf{1}_{E_{1}}\right]$ may not equal $p_{1} \mathrm{E}\left[\alpha^{\tau_{\mathcal{C}}}\right]$.

Nevertheless, if the $i$ th, $i \leq K$, scenario occurs, then we know the value of $\tau_{\mathcal{C}}$ exactly. It is equal to $\left|A_{i}\right|$, the length of the $i$ th sequence. Therefore, we have a formula for the stopped martingale:

$$
X_{\tau_{\mathcal{C}}}=\frac{\alpha^{2}-\alpha \alpha^{\tau}}{1-\alpha} \sum_{j=1}^{L+2 M} y_{j}^{*}-\alpha^{\tau}-I\left(\alpha, y_{1}^{*}, \ldots, y_{L+2 M}^{*}\right)
$$

Here, $I\left(\alpha, y_{1}^{*}, \ldots, y_{L+2 M}^{*}\right)$ is defined by setting

$$
I\left(\alpha, y_{1}^{*}, \ldots, y_{L+2 M}^{*}\right)= \begin{cases}\alpha^{\left|A_{i}\right|}\left[\sum_{j=1}^{L+2 M} y_{j}^{*} W_{i j}(\alpha)-1\right] & \text { in scenario } i \in\{1,2, \ldots, K\} \\ 0, & \text { in scenario } i>K .\end{cases}
$$

From this formula and the optional stopping theorem, we then find the anticipated formula for the moment generating function of $\tau_{\mathcal{C}}$.

Theorem 2. If $\left(y_{1}^{*}, \ldots, y_{L+2 M}^{*}\right)$ is a solution to the linear system (4), then

$$
\mathrm{E}\left[\alpha^{\tau_{\mathcal{C}}}\right]=\frac{\left[\alpha^{2} /(1-\alpha)\right] \sum_{j=1}^{L+2 M} y_{j}^{*}-\sum_{i=1}^{K} p_{i} \alpha^{\left|A_{i}\right|}\left[\sum_{j=1}^{L+2 M} y_{j}^{*} W_{i j}-1\right]}{1+[\alpha /(1-\alpha)] \sum_{j=i}^{L+2 M} y_{j}^{*}} .
$$

\subsection{Computation of the $\alpha$-profit matrix}

As before, we need to know how to compute the profit matrix before (5) may be properly regarded as an explicit formula. This is only a little more difficult than before. First, assume 
that a scenario ends with the pattern $C=c_{1} c_{2} \cdots c_{m}$. The team of straightforward gamblers who bet a dollar on pattern $A=a_{1} a_{2} \cdots a_{p}$ will, by time $\tau$, win an amount

$$
\sum_{i=1}^{\min \{m-1, p\}} \frac{\delta_{i}^{\text {st }}(A, C)}{\alpha^{i-1}}
$$

while, in the same time, the team of smart gamblers will win an amount

$$
\sum_{i=1}^{\min \{m-1, p\}} \frac{\delta_{i}^{\mathrm{sm} 1}(A, C)}{\alpha^{i-1}}+\sum_{i=1}^{\min \{m-1, p-1\}} \frac{\delta_{i}^{\mathrm{sm} 2}(A, C)}{\alpha^{i-1}}
$$

These formulae provide almost everything we need, but before we can be completely explicit, we need to focus on a concrete example.

\subsection{A generating function example}

Consider the waiting time to observation of the three-letter pattern FSF in the random sequence $\left\{Z_{n}, n \geq 1\right\}$ produced by the Markov model. In this case, the $\alpha$-profit matrix $\left\{W_{i j}(\alpha)\right\}$ is given by

$$
\left(\begin{array}{cc}
\frac{1}{p_{S F}} & \frac{\alpha^{-1}}{p_{F S} p_{S F}}+\frac{1}{p_{S F}} \\
\frac{\alpha^{-2}}{p_{S F} p_{F S} p_{S F}}+\frac{1}{p_{S F}} & \frac{\alpha^{-2}}{p_{S F} p_{F S} p_{S F}}+\frac{\alpha^{-1}}{p_{F S} p_{S F}}+\frac{1}{p_{S F}} \\
\frac{\alpha^{-2}}{p_{F F} p_{F S} p_{S F}}+\frac{1}{p_{S F}} & \frac{\alpha^{-1}}{p_{F S} p_{S F}}+\frac{1}{p_{S F}}
\end{array}\right),
$$

and, by solving the associated linear system, we obtain

$$
y_{1}^{*}=\frac{\alpha^{2} p_{F F} p_{F S} p_{S F}}{1-\alpha p_{F F}+\alpha p_{S F}+\alpha^{2} p_{F S} p_{S F}}, \quad y_{2}^{*}=\frac{\alpha^{2} p_{F S} p_{S F}\left(p_{S F}-p_{F F}\right)}{1-\alpha p_{F F}+\alpha p_{S F}+\alpha^{2} p_{F S} p_{S F}} .
$$

The general moment generating representation (5) then gives us the simple formula

$$
\mathrm{E}\left[\alpha^{\tau_{\mathcal{C}}}\right]=\frac{\alpha^{3} p_{F S} p_{S F}\left(p_{F}+\alpha\left(p_{S}-p_{S S}\right)\right)}{1-\alpha\left(p_{S S}+p_{F F}-\alpha\left(p_{F F}-p_{S F}\left(1-p_{F S}\left(1-\alpha p_{S S}\right)\right)\right)\right)} .
$$

Naturally, such a formula provides complete information on the distribution of $\tau_{\mathcal{C}}$, and to obtain an explicit formula for $\mathrm{P}\left(\tau_{\mathcal{C}}=k\right)$ we can rewrite the rational function (5) in its partial fraction expansion.

\section{Higher-order Markov chains}

In this work, we have applied the gambling team method only to two-state chains. For reasons that will be explained later, this limitation is not easily lifted. Nevertheless, there are more complex chains to which the team method applies, and it is instructive to consider one of these. Specifically, we briefly consider how the gambling team method may be applied to second-order two-state chains. Here we obviously need to avoid the naive representation of such chains as first-order chains with four states.

In the team approach to a second-order model, the gamblers need to observe two rounds of betting before they place their first bets, and, consequently, we need to consider a larger number 
of final scenarios. Moreover, for each pattern $A=a_{1} a_{2} \cdots a_{p}$ we will need to consider up to seven termination cases, including three 'initial' cases, which are associated with the patterns $A, S A$, and $F A$, and four 'later' cases, which are associated with the patterns SSA, SFA, FSA, and FFA.

As before, our main objective is to count accurately all the ending scenarios and create a matched number of gambling teams. However, for a second-order chain there is an additional difficulty that must be addressed. Specifically, two cases must be considered separately: (i) there are no runs in the initial list $\mathcal{C}$ and (ii) there are runs in $\mathcal{C}$.

\subsection{Case one: there are no runs in $\mathcal{C}$}

First we need to replace the earlier doubling step with an analogous quadrupling step. Now, given the collection of patterns $\mathcal{C}=\left\{A_{i}\right\}_{1 \leq i \leq K}$, we consider the set sequence transformation

$$
\mathcal{C}=\left\{A_{i}\right\}_{1 \leq i \leq K} \rightarrow\left\{S S A_{i}, S F A_{i}, F S A_{i}, F F A_{i}\right\}_{1 \leq i \leq K}=\left\{B_{i}\right\}_{1 \leq i \leq 4 K}=\mathcal{C}^{\prime} .
$$

We then delete from $\mathcal{C}^{\prime}$ each scenario that can occur only after the stopping time $\tau_{\mathcal{C}}$, and we take the collection $\mathcal{C}^{\prime \prime}$ that remains to be our 'final list' of ending scenarios.

Each pattern from the collection $\mathcal{C}$ leads us to four - or perhaps fewer - ending scenarios. For each pattern from $\mathcal{C}$ we consider a sequence of gamblers who belong to teams of different types. As before, these gamblers arrive sequentially and observe the game before placing any bets, according to the following rules.

1. A new gambler from the 'type-I' team arrives two rounds before he begins to bet. He watches these rounds and then bets on the successive letters of pattern $A$, with complete indifference to what he may have seen in the first two rounds he observed.

2. A new gambler from the 'type-II' team also arrives two rounds before he begins to gamble, but is influenced by what he sees. If this gambler observes the pattern $S a_{1}$ in these two rounds, then he bets on the sequence $a_{2} a_{3} \cdots a_{p}$. If he observes anything other than $S a_{1}$, then he places his bets according to the sequence $A$.

3. Similarly, a new gambler from the 'type-III' team watches two rounds, and if he observes the pattern $F a_{1}$ then he bets according to the sequence $a_{2} a_{3} \cdots a_{p}$. If he observes anything other than $F a_{1}$, then he places his bets according to $A$.

4. Finally, a gambler from the 'type-IV' team watches two rounds, and if he observes $a_{1} a_{2}$ then he bets according to the sequence $a_{3} a_{4} \cdots a_{p}$. Otherwise, he bets according to the sequence $A$.

Each pattern $A$ from the initial list $C$ leads to zero, one, two, three, or four scenarios in the final list $\mathcal{C}^{\prime \prime}$. Thus, instead of there being only matched and unmatched patterns, the patterns in final list $\mathcal{C}^{\prime \prime}$ are now of four kinds: 'unmatched,' 'double-matched,' 'triple-matched,' and 'quadruple-matched.'

To see how this works, consider the initial collection $\mathcal{C}=\{F S F F, F F S F\}$. First, note that we have five initial cases, namely FSFF, FFSF, SFSFF, SFFSF, and FFFSF. Pattern FFSFF cannot occur before $\tau_{\mathcal{C}}$; therefore, the scenario associated with this pattern is eliminated from the list of initial cases. Second, in the final list $\mathcal{C}^{\prime \prime}$ we have five patterns: the double-matched patterns SSFSFF and FSFSFF generated by FSFF, and the triple-matched patterns SSFFSF, $S F F F S F$, and $F F F F S F$ generated by $F F S F$. Thus, we need to introduce five teams: type-I and type-II teams that bet on FSFF, and type-I, type-II, and type-III teams that bet on FFSF. 


\subsection{Case two: special treatment of runs}

If the initial list $\mathcal{C}$ contains a run, then there may be a problem with the straightforward application of the method described above. The difficulty is that if we observe the game only until the moment $\tau_{\mathcal{C}}$, then there is no difference in behavior between teams of different types that place their bets on the run.

To illustrate the problem, let us consider the initial list $\mathcal{C}=\left\{F^{(r)}\right\}$. The straightforward usage of the above algorithm tells us that we must introduce two initial cases, $F^{(r)}$ and $S F^{(r)}$. The final list $\mathcal{C}^{\prime \prime}$ contains two double-matched patterns, $S S F^{(r)}$ and $F S F^{(r)}$. Therefore, according to the algorithm, we need two (type-I and type-II) teams that respectively bet $y_{1}$ and $y_{2}$ dollars on $F^{(r)}$. However, since before time $\tau_{\mathcal{C}}$ there is no difference in gambling between these two teams, we in effect have only one team, which bets $y_{1}+y_{2}$ dollars on the run. Thus, the number of free parameters does not match the number of ending scenarios.

However, a simple modification of the gambling method easily solves the problem. Before time $\tau_{\mathcal{C}}$, the run $F^{(r)}$ can only occur as an initial segment of the sequence $\left\{Z_{n}, n \geq 1\right\}$, or, later, in the pattern $S F^{(r)}$. Thus, if the initial list $\mathcal{C}$ contains runs (obviously we can have only one or two runs in $\mathcal{C}$, namely $F^{(r)}$ or $S^{(p)}$ or both), then we first need to substitute the runs $F^{(r)}$ and $S^{(p)}$ in $\mathcal{C}$ by $S F^{(r)}$ and $F S^{(p)}$, respectively, to obtain a different collection $\tilde{\mathcal{C}}$. The collection $\tilde{\mathcal{C}}$ contains no runs; therefore, we can proceed as before. After application of the quadrupling and elimination processes to the list $\tilde{\mathcal{C}}$ we will obtain the final list of ending scenarios $\tilde{\mathcal{C}}^{\prime \prime}$, and for this list we will be able to create a matched number of gambling teams. Since we are interested in $\tau_{\mathcal{C}}$, not $\tau_{\tilde{\mathcal{C}}}$, the elimination process has to be based on $\tau_{\mathcal{C}}$, and the runs must be included in the list of initial cases.

For instance, if $\mathcal{C}=\left\{F^{(r)}\right\}$ then we must consider four initial cases, $F^{(r)}, S F^{(r)}, S S F^{(r)}$, and $F S F^{(r)}$, and four later cases, in which the game ends with $\operatorname{SSSF}^{(r)}, \operatorname{SFSF}^{(r)}, \operatorname{FSSF}^{(r)}$, or $F F S F^{(r)}$. For this collection, it can be shown that each of the four teams (type I, II, III, and IV) that bet on $S F^{(r)}$ bets in its own way.

\subsection{Final step}

After attending to this bookkeeping, we can now calculate the expected observation times in a way that parallels our earlier calculation. Since we have matched the number of (non-initial) ending scenarios and the number of teams, we can choose the size of the initial bet for each team in a way that makes all the expressions for the stopped martingale equal to 1 , however the game may end.

Let us summarize this as a theorem. Assume that ultimately we have $P$ initial cases and $Q$ later cases. Let $W_{i j} y_{j}, i=1,2, \ldots, P+Q, j=1,2, \ldots, Q$, denote the amount of money won by the $j$ th team that bets $y_{j}$ dollars in the $i$ th scenario. Finally, let $p_{i}, i=1,2, \ldots, P$, be the probability that the $i$ th initial scenario occurs.

Theorem 3. If $\left(y_{1}^{*}, y_{2}^{*}, \ldots, y_{Q}^{*}\right)$ solves the linear system

$$
\begin{aligned}
y_{1}^{*} W_{P+1,1}+\cdots+y_{Q}^{*} W_{P+1, Q} & =1, \\
\vdots & \vdots \\
y_{1}^{*} W_{P+Q, 1}+\cdots+y_{Q}^{*} W_{P+Q, Q} & =1,
\end{aligned}
$$

then

$$
\mathrm{E}\left[\tau_{\mathrm{C}}\right]=2+\frac{\sum_{i=1}^{P} p_{i} \sum_{j=1}^{Q} y_{j}^{*} W_{i j}+\left(1-\sum_{i=1}^{P} p_{i}\right)}{\sum_{j=1}^{Q} y_{j}^{*}} .
$$




\section{Concluding remarks}

The method of gambling teams deals quite effectively with the waiting time problems of twostate chains, but for $N$-state chains it is much less effective. The problem is that we typically find that the number of ending scenarios is larger than the number of teams, meaning that there are too few free parameters to achieve the required matching.

We might think to reduce the waiting time problems for an $N$-state chain by encoding the states $\{1,2, \ldots, N\}$ as sequences of 0 s and $1 \mathrm{~s}$, but this idea typically fails, since the natural encodings do not lead to a waiting time problem for a homogeneous two-state Markov chain on $\{0,1\}$. Ironically, for many of the pattern problems associated with $N$-state Markov chains, the method of gambling teams is ineffective when $N \geq 3$, even though it is typically the method of choice for the corresponding problems in a two-state chain.

A possible computational advantage of the martingale method over the Markov chain embedding method (see, e.g. Antzoulakos (2001), Fu (2001), and Fu and Chang (2002)) is the size of the matrices involved in the calculations. The size of the profit matrix depends only on the number of patterns, $K$, while the size of the transition matrix of the embedded Markov chain depends also on the length of patterns in $\mathcal{C}$. For example, if $\mathcal{C}$ contains $K$ patterns, each of which has a length that is approximately $N$, and $K$ is much smaller than $N$, then the dimensions of the transition matrix in the Markov chain embedding method are approximately $(K \times N) \times(K \times N)$. For large $N$, this can cause technical problems. The size of profit matrix is at most $2 K \times 2 K$.

\section{References}

Aki, S., Balakrishnan, N. And Mohanty, S. G. (1996). Sooner and later waiting time problems and failure runs in higher order Markov dependent trials. Ann. Inst. Statist. Math. 48, 773-787.

Antzoulakos, D. (2001). Waiting times for patterns in a sequence of multistate trials. J. Appl. Prob. 38, 508-518.

Benevento, R. V. (1984). The occurrence of sequence patterns in ergodic Markov chains. Stoch. Process. Appl. 17, 369-373.

Biggins, J. D. AND CANnings, C. (1987a). Formulas for mean restriction-fragment lengths and related quantities. Amer. J. Hum. Genet. 41, 258-265.

Biggins, J. D. And Cannings, C. (1987b). Markov renewal processes, counters and repeated sequences in Markov chains. Adv. Appl. Prob. 19, 521-545.

Blom, G. AND Thorburn, D. (1982). How many random digits are required until given sequences are obtained? J. Appl. Prob. 19, 518-531.

Breen, S., Waterman, M. and Zhang, N. (1985). Renewal theory for several patterns. J. Appl. Prob. 22, $228-234$.

Chryssaphinou, O. AND PAPAstavridis, S. (1990). The occurrence of a sequence of patterns in repeated dependent experiments. Theory Prob. Appl. 35, 145-152.

Feller, W. (1968). An Introduction to Probability Theory and Its Applications, Vol. 1, 3rd edn. John Wiley, New York.

FU, J. C. (1986). Reliability of consecutive- $k$-out-of- $n$ : $F$ systems with $(k-1)$-step Markov dependence. IEEE Trans. Reliab. 35, 602-606.

FU, J. C. (2001). Distribution of the scan statistics for a sequence of bistate trials. J. Appl. Prob. 38, 908-916.

FU, J. C. AND CHANG, Y. (2002). On probability generating functions for waiting time distribution of compound patterns in a sequence of multistate trials. J. Appl. Prob. 39, 70-80.

Fu, J. C. And Koutras, M. V. (1994). Distribution theory of runs: a Markov chain approach. J. Amer. Statist. Assoc. 78, 168-175.

Gerber, H. AND LI, S. (1981). The occurrence of sequence patterns in repeated experiments and hitting times in a Markov chain. Stoch. Process. Appl. 11, 101-108.

Guibas, L. And OdlyzKo, A. (1981a). Periods of strings. J. Combinatorial Theory A 30, 19-42.

Guibas, L. AND OdLYzKo, A. (1981b). String overlaps, pattern matching and nontransitive games. J. Combinatorial Theory A 30, 183-208.

Han, Q. And Hirano, K. (2003). Sooner and later waiting time problems for patterns in Markov dependent trials. J. Appl. Prob. 40, 73-86.

LI, S. (1980). A martingale approach to the study of occurrence of sequence patterns in repeated experiments. Ann. Prob. 8, 1171-1176. 
Pozdnyakov, V. ANd KulldorfF, M. (2006). Waiting times for patterns and a method of gambling teams. Amer. Math. Monthly 113, 134-143.

Pozdnyakov, V., Glaz, J., Kulldorff, M. and Steele, J. M. (2005). A martingale approach to scan statistics. Ann. Inst. Statist. Math. 57, 21-37.

Robin, S. And Daudin, J.-J. (1999). Exact distribution of word occurrences in a random sequence of letters. J. Appl. Prob. 36, 179-193.

Stefanov, V. T. (2000). On some waiting time problems. J. Appl. Prob. 37, 756-764.

Stefanov, V. T. (2003). The intersite distances between pattern occurrences in strings generated by general discreteand continuous-time models: an algorithmic approach. J. Appl. Prob. 40, 881-892.

Stefanov, V. T. And Pakes, A. G. (1997). Explicit distributional results in pattern formation. Ann. Appl. Prob. 7, $666-678$.

UCHIDA, M. (1998). On generating functions of waiting time problems for sequence patterns of discrete random variables. Ann. Inst. Statist. Math. 50, 655-671.

Williams, D. (1991). Probability with Martingales. Cambridge University Press. 